\title{
El estrés oxidativo en la fertilidad y desempeño reproductivo de mamíferos hembras y machos
}

\author{
C.Izquierdo, A.'; Iglesias, A.E. ${ }^{2}$; Guerra, J.E. ${ }^{3}$; Huerta, R. ${ }^{4}$; Sánchez, R. ${ }^{5}$ \\ ${ }^{1}$ Dep. Prod. Agríc. Anim., Univ.Aut. Metrop. Xochimilco, México. ${ }^{2}$ Maestría Cs. Agrop. Univ. Autón. \\ Xochimilco. ${ }^{3} \mathrm{Fac}$. Agron. Univ. Sinaloa, México. ${ }^{4} \mathrm{Fac}$. Vet. \\ Univ. Autón. Puebla, México. ${ }^{5}$ Dep.Reprod. INIA, Madrid, España. \\ E-mail: acordova@correo.xoc.uam.mx
}

\begin{abstract}
Resumen
C.Izquierdo, A.; Iglesias, A.E.; Guerra, J.E.; Huerta, R.; Sánchez, R.: El estrés oxidativo en la fertilidad y desempeño reproductivo de mamíferos hembras y machos. Rev. Vet. 31: 1, 97-100, 2020. Los radicales libres son agentes de oxidación altamente reactivos, que actúan como intermediarios químicos de vida corta sobre lípidos, aminoácidos, carbohidratos y ácidos nucleicos. Cuando ocurren desequilibrios y la cantidad de radicales libres sobrepasa el balance entre la producción oxidante y la capacidad antioxidante en la célula, se genera un fenómeno conocido como estrés oxidativo. Tal anomalía genera consecuencias negativas sobre múltiples procesos celulares de los mamíferos, tanto en las hembras como en los machos, y -por lo tanto- daños en la fertilidad y desempeño reproductivo. En esta revisión se describe brevemente el estrés oxidativo en la membrana plasmática, las mitocondrias, el acrosoma y el ADN de los espermatozoides, así como el efecto del estrés oxidativo sobre la fertilidad de machos y hembras.
\end{abstract}

Palabras clave: mamíferos, estrés oxidativo, fertilidad, hembra, macho.

\begin{abstract}
C.Izquierdo, A.; Iglesias, A.E.; Guerra, J.E.; Huerta, R.; Sánchez, R.: Oxidative stress in fertility and reproductive performance of females and males mammals. Rev. Vet. 31: 1, 97-100, 2020. Free radicals are highly reactive oxidation agents, which act as short-lived chemical intermediates on lipids, amino acids, carbohydrates and nucleic acids. When there is an imbalance and the amount of free radicals exceeds the balance between oxidant production and antioxidant capacity in the cell, a phenomenon known as oxidative stress is generated, which has negative consequences on multiple cellular processes, both in females as in males and therefore negative effect on fertility and reproductive performance. In this work, the oxidative stress in the spermatozoa, plasma membrane, mitochondria, acrosome and DNA is briefly described, as well as the effect of oxidative stress on female and male fertility.
\end{abstract}

Key words: mammals, oxidative stress, fertility, female, male.

\section{Introducción}

En las áreas de las medicinas veterinaria y humana se están descubriendo cada vez más agentes causantes de enfermedades, algunos de ellos derivados de procesos metabólicos del oxígeno tales como la producción de energía, la detoxificación de compuestos nocivos y la defensa frente a los agentes patógenos. Dentro de esta última figuran los radicales libres (RL), agentes de oxidación altamente reactivos que actúan como intermediarios químicos de vida corta sobre lípidos, aminoácidos, carbohidratos y ácidos nucleicos ${ }^{8}$.

Los RL pueden ser especies reactivas de oxígeno (ROS), las cuales son moléculas altamente reactivas que atacan constantemente al organismos mediante reacciones de óxido-reducción. Dentro de este grupo

Recibido: febrero 2020 / Aceptado: abril 2020 se encuentran el oxígeno molecular $\left(\mathrm{O}_{2}\right)$, el anión superóxido $\left(\mathrm{O}_{2}{ }^{1}\right)$ el peróxido de hidrógeno $\left(\mathrm{H}_{2} \mathrm{O}_{2}\right)$, el hidroperoxilo $\left(\mathrm{HO}_{2}\right)$ y el radical hidroxilo $(\mathrm{OH})$.

Otros RL son los metales de transición que poseen electrones desapareados y también las especies reactivas de nitrógeno, que son capaces de generar daño oxidante y muerte celular. Dentro de este grupo se encuentran el óxido nítrico $(\mathrm{ON})$, el anión peroxi-nitrito y el dióxido nítrico ${ }^{5}$.

Los RL deben ser atenuados por diferentes sistemas de defensa antioxidante, que involucran enzimas y moléculas. Los antioxidantes se dividen en enzimáticos y no enzimáticos. Los primeros, también llamados de producción endógena, constituyen la línea de defensa inicial frente a la producción de RL. Son proteínas con capacidad antioxidante que no se consumen al reaccionar con los RL, tales como catalasa, superóxido dismutasa y glutatión peroxidasa. 
En cambio, los antioxidantes no enzimáticos provienen principalmente de la dieta y son pequeñas moléculas liposolubles, que a diferencia de los enzimáticos se consumen durante su acción antioxidante, por lo cual deben ser remplazados. Entre los más importantes de este grupo figuran las vitaminas E, C, beta carotenos, retinol, ácido úrico, piruvato, albúmina, carnitina, taurina, hipotaurina, transferrina, ceruloplasmina, polifenoides, flavonoides y oligoelementos ${ }^{6}$.

Las vías de defensa antioxidante se encuentran enlazados en un sistema amortiguador celular, donde se suman y colaboran entre sí, para hacer frente a cualquier agresión oxidativa en las células. Entre ellos, los antioxidantes no enzimáticos pueden tener efectos sinérgicos en combinación con los antioxidantes enzimáticos, regenerando estos últimos mediante la donación de hidrogeno, neutralizando el oxígeno molecular y catalizando la síntesis o la regeneración de antioxidantes no enzimáticos.

Cuando ocurre un desequilibrio y la cantidad de RL sobrepasa el balance entre la producción oxidante y la capacidad antioxidante, se genera un fenómeno conocido como estrés oxidativo (EO), el cual tiene consecuencias negativas sobre múltiples procesos celulares, tanto en las hembras como en los machos, incluyendo efectos nocivos para la fertilidad y el desempeño reproductivo $^{8}$.

\section{Efectos del estrés oxidativo en los espermatozoides}

Cuando existe EO en el macho, las ROS afectan principalmente a componentes de los espermatozoides tales como proteínas, ácidos nucleicos, azúcares, lípidos y mitocondrias ${ }^{8}$.

La mayor parte de las principales enfermedades que provocan la muerte de animales y personas o deterioran su calidad de vida, son provocadas por los RL. Cada célula del cuerpo padece unos 10.000 impactos de radicales libres por día, motivo por el cual el EO ha sido objeto de intensas investigaciones en años recientes.

Las enfermedades crónicas degenerativas que se pueden presentar en los seres humanos son cáncer, osteoporosis, Alzheimer, diabetes tipo 2, padecimientos neuro-degenerativos y enfermedades cardiovasculares como insuficiencia cardiaca ${ }^{8}$.

Los espermatozoides fueron las primeras células en las cuales se pudo identificar la presencia de ROS, ya que hasta hace pocos años, eran consideradas elementos tóxicos para los espermatozoides. Sin embargo, en la actualidad se sabe que los RL (principalmente el $\mathrm{O}_{2}$ ) en bajas concentraciones en el semen, cumplen un papel fundamental en sus funciones biológicas durante la capacitación y maduración espermática, la fosforilación de tirosina, la interacción inter-gametos y la reacción acrosomal que se da para la fecundación del ovocito. Por lo tanto, está relacionado con la fertilidad y el desempeño reproductivo de seres humanos y animales.
Tales fenómenos están controlados por mecanismos de defensa de antioxidantes enzimáticos y no enzimáticos. Cuando se quiebra el equilibrio entre los RL y el sistema de defensa antioxidante, se inducen daños en los ácidos nucleicos, las proteínas y los lípidos presentes en la membrana de los espermatozoides, causando pérdida de la movilidad, disminución en la viabilidad y alteraciones en la pieza intermedia, anomalías que finalmente producen disminución de la calidad seminal o muerte espermática ${ }^{1,4}$.

Otro ejemplo claro es el efecto del ON, el cual ejerce una función importante en la fisiopatología espermática, ya que en bajas concentraciones favorece los procesos de capacitación espermática, la reacción acrosomal y la unión a la zona pelúcida. Sin embargo, en concentraciones elevadas conduce a la formación de peroxi-nitritos que alteran la motilidad espermática ${ }^{4}$.

Se ha podido constatar que en el eyaculado, las principales fuentes de ROS son los leucocitos y las células espermáticas anormales, aunque se ha propuesto que existen otras posibilidades sobre la generación de ROS intracelular en el espermatozoide, como es la fuga de electrones de la cadena de transporte mitocondrial, la NADPH-oxidasa como una posible fuente de ROS y la generación de RL por medio de óxido nítrico en las regiones post-acrosomales y ecuatoriales, que pueden generar cambios del estado basal de los agentes oxidantes e inducir trastornos de la actividad espermática ${ }^{8}$.

\section{Efectos en la membrana citoplasmática}

La membrana espermática es asimétrica en su estructura y en sus funciones. Está formada por una asociación de fosfolípidos, plasmalógenos y esfingomielinas en equilibrio dinámico con proteínas de membrana, convirtiéndola en un blanco fácil para el ataque de los agentes oxidantes. El colesterol y los fosfolípidos son importantes en el mantenimiento de la integridad estructural de los sistemas de membrana.

En particular, la membrana plasmática de los espermatozoides posee gran cantidad de ácidos grasos poliinsaturados, necesarios para la reacción acrosomal y la interacción con la membrana del ovocito. Por otra parte, el elevado contenido de ácidos grasos poli-insaturados de las membranas plasmáticas de los espermatozoides, los torna muy susceptibles a sufrir lipo-peroxidación, haciéndolas altamente vulnerables al estrés oxidante ${ }^{8,11}$.

Las bajas concentraciones de enzimas antioxidantes en la membrana plasmática (catalasas, dismutasas, peroxidasas, glutatión reductasa), también tienden a convertir los espermatozoides en células susceptibles al ataque de los RL, particularmente a la agresión del radical hidroxilo $\mathrm{OH}$ y del hidro-peroxilo $\left(\mathrm{HO}_{2}\right)$, sobre todo en la región post-acrosomal.

Ello ocasiona alteraciones en su permeabilidad, ya que las ROS inducen lipo-peroxidación de los fosfolípidos de la membrana, y ello provoca la aparición de "orificios" afectando a las bombas de $\mathrm{Na}^{+} \mathrm{y} \mathrm{Ca}^{2+}$, produciendo la entrada de estos cationes al interior del 
espermatozoide, alterando su osmolaridad. Ello causa la formación de fosfatos de calcio poco solubles, el agotamiento del ATP y la activación por medio del $\mathrm{Ca}^{2} \mathrm{de}$ las enzimas proteolíticas y fosfoglucolipídicas, en pocas palabras, aumentando su permeabilidad.

También daña a las enzimas lactato-deshidrogenasa, piruvato-kinasa, gliceraldehido-3-fosfato-deshidrogenasa y ATPasa, generando la pérdida o reducción de la movilidad, daño proteico y lipídico, alteraciones en el ácido desoxi-ribonucleico (ADN), anomalías en su morfología, problemas de fertilidad y muerte celular ${ }^{11}$.

\section{Efectos en el acrosoma}

El acrosoma también se ve afectado por la acción de los RL durante el transporte de los espermatozoides a través del epidídimo y -mayormente- después de ser eyaculados, principalmente por el peróxido de hidrógeno. Este último inhibe la inducción de la reacción acrosomal y daña la integridad del acrosoma, produciendo un mal funcionamiento al momento de la fecundación del ovocito, cuyo resultados es la baja fertilidad reflejada tanto en hembras como en machos, por lo cual las ganancias del productor ganadero son menores ${ }^{3}$.

\section{Efecto en las mitocondrias}

Las mitocondrias son consideradas como una de las principales fuentes celulares atacadas por las ROS, ya que su función es la regulación de procesos fisiológicos como la transducción de señales intracelulares, la respuesta al estrés oxidante, el desarrollo embrionario, la proliferación y adhesión celular, la expresión genética y la apoptosis ${ }^{8}$.

En los espermatozoides las mitocondrias proveen la mayor cantidad de ATP, por medio de la glucólisis y fosforilación oxidativa, contribuyendo a la formación de RL durante estos procesos ${ }^{8}$. Sin embargo, cuando existe interrupción de la cadena respiratoria mitocondrial (p.ej. durante la congelación), ellas son las responsables de la formación y liberación de ROS.

Tal interrupción provoca que el oxígeno sufra reducciones completas produciendo -en lugar de moléculas de agua- moléculas intermedias como el anión superóxido, el radical hidroxilo y el peróxido de hidrógeno, desencadenando un efecto similar a la apoptosis, fenómeno responsable tanto de la muerte de los espermatozoides, como de los daños sub-letales que disminuyen su vida media y capacidad fecundante, así como el desempeño reproductivo de los machos (Figura 1) ${ }^{9}$.

\section{Efectos en el ADN}

Gran parte del daño del ADN en los espermatozoides es generado por el EO. Los daños que las ROS ejercen directamente sobre el ADN espermático, pueden inducir mutaciones, afectando la genómica paterna del embrión, daño que puede ser considerado como indicativo de infertilidad masculina ${ }^{11}$.

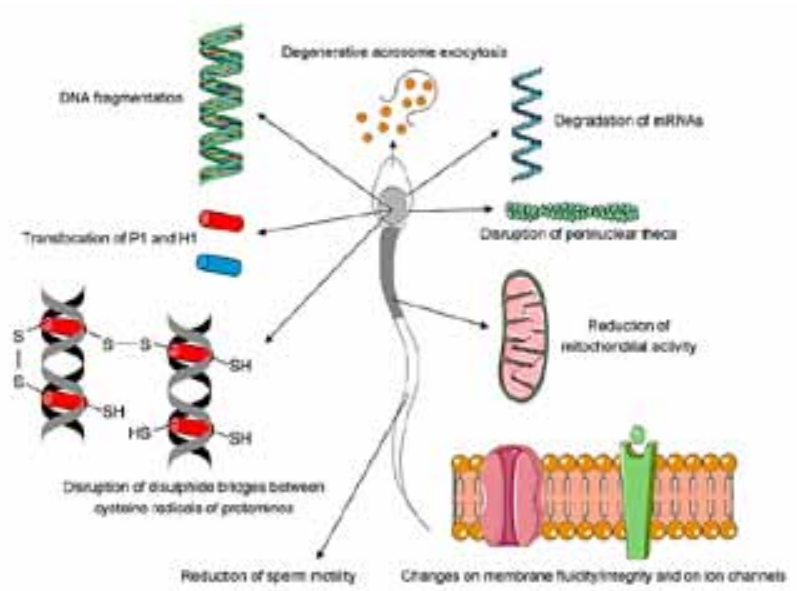

Figura 1. Lesiones resultantes de la congelación de semen del cerdo ${ }^{14}$ modificado .

Para demostrar lo anterior, espermatozoides fueron expuestos a altas concentraciones de ROS producidas artificialmente, generando como resultado el aumento significativo en el daño del ADN, disminución en la motilidad espermática e inducción de procesos apoptóticos (muerte programada de los espermatozoides) ${ }^{8}$.

Tales daños espermáticos en testículos o epidídimo dependen de factores endógenos (durante la maduración espermática) y exógenos como el daño peroxidativo del ADN, infecciones, factores inmunológicos y diversos agentes químicos. Además, pueden estar relacionados con fallas en el empaquetamiento, la madurez nuclear, la fragmentación de la cromatina, aneuploidías o defectos en la integridad del $\mathrm{ADN}^{8,11}$.

Es importante destacar que principalmente existen dos RL que afectan la hebra de ADN. Uno de ellos es el radical $\mathrm{OH}$, que resulta en la formación de $8-\mathrm{OH}$-guanina y 8-OH-2-deoxiguanosina en un primer estadio, atacando las purinas y pirimidinas, ocasionando fragmentación del ADN de cadena doble. El segundo es el radical $\mathrm{O}_{2}$, que generalmente produce solo aductos de guanina, sobre todo 8-hidroxi-guanina, afectando la motilidad espermática ${ }^{14}$.

Si un espermatozoide con fragmentación de ADN logra fecundar un ovocito, la fertilización es inviable, resultando anormal el desarrollo de la gestación ${ }^{11}$.

\section{Efectos en la fertilidad de hembras y machos}

En seres humanos, la infertilidad se define como la incapacidad de una pareja para tener hijos luego de un año de relaciones sexuales sin medidas anti-conceptivas ${ }^{11}$. Existen múltiples causas de infertilidad masculina, pudiendo ser congénitas o adquiridas. Entre todas ellas, la infertilidad idiopática está causada por múltiples factores como alteraciones endocrinas, estrés oxidante, fallas genéticas o epigenéticas y otras ${ }^{10}$.

En animales, la infertilidad es definida como la incapacidad de reproducirse o tener crías. La anomalía puede ser temporal o indefinida y está relacionada a una infinidad de factores, entre ellos fallas genéticas, 
insalubridad y malas condiciones corporales, tanto en hembras como en machos.

En personas, el papel del EO como una de las principales causas de infertilidad masculina ha sido bien establecido, ya que las ROS pueden afectar todos los componentes celulares de los espermatozoides, como ya se ha indicado ${ }^{11}$.

Es importante destacar la importancia de los antioxidantes en el semen, ya que se ha observado que los bajos niveles o deficiencia de antioxidantes en el plasma seminal, dejan a los espermatozoides desprotegidos contra el EO ${ }^{13}$. Por ello, se ha propuesto el uso de antioxidantes como herramienta para proteger a los espermatozoides del daño oxidativo; incluso se ha comprobado que la adición de antioxidantes (vitamina $\mathrm{C}$, E o glutatión) a la hora de la conservación del semen, producen mejores resultados en la evaluación seminal al momento de la inseminación ${ }^{2,12,14}$.

Para el caso de la mujer, se ha planteado que las ROS pueden participar en la formación de adherencias asociadas con endometriosis, disminuyendo su fertilidad. También existen alteraciones de la foliculogénesis causadas por las ROS, que pueden deteriorar la calidad del ovocito, habiéndose propuesto como una causa de subfertilidad asociada con endometriosis.

En los animales domésticos, el EO también se ha asociado a numerosas patologías entre las que pueden mencionarse: mastitis, edema de la ubre, mayor incidencia de enfermedades en el período periparto, déficit en la síntesis de hormonas esteroides en vacas y miopatía nutricional degenerativa en ovinos. En el caso de las cerdas, el EO puede ocasionar estados inflamatorios post-destete, modificando el estatus de selenio y vitamina $\mathrm{E}$, afectando la tasa de crecimiento de los lechones ${ }^{7}$.

\section{A manera de conclusión}

El estrés oxidativo afecta de manera importante la capacidad fecundante de los espermatozoides de mamíferos, ocasionando infertilidad en los machos y/o bajos parámetros reproductivos en las hembras. Tales anomalías afectan significativamente las unidades de producción animal, por lo cual constituyen un tema de gran importancia en la actualidad.

Agradecimientos. Al Consejo Nacional de Ciencia y Tecnología (CONACYT, México), por el apoyo brindado.

\section{REFERENCIAS}

1. Álvarez RM, Carrillo VA, Rodríguez MH. 2017. Exogenous individual lecthin-phospholipids (phosphatidylcholine and phosphatidylglycerol) cannot prevent the oxidative stress imposed by criopreservation of boar sperm. $J$ Vet Med Surg 2: 1-11.

2. Córdova IA. 2010. Control de la peroxidación lipídica del semen refrigerado y criopreservado de verraco mediante antioxidantes y su repercusión sobre la calidad espermática. Tesis Doctoral. Univ. León, España, p. 1-290.

3. Córdova IA et al. 2017. Effect of addition of antioxidants in the extender to freeze boar in two types of straws on sperm quality. Int J Rec Sci Res 8: 17466-17468.

4. Espinosa CR, Córdova IA. 2018. Efecto del estrés calórico y el estrés oxidativo en la función espermática de los mamíferos. Rev Compl Cs Vet 12: 27-39.

5. García TB, Saldaña BA, Saldaña GL. 2012. El estrés oxidativo y los antioxidantes en la prevención del cáncer. Rev Hab Cs Méd 12: 187-196.

6. Gumbao BD. 2015. Efecto antioxidante del glutatión aplicado en el medio de descongelación seminal de tres especies con interés. Anales Univ. Murcia 1-154.

7. Gupta S. 2010. Mecanismos patogénicos de la infertilidad asociada con endometriosis. Rev Mex Med Reprod 3: 83-97.

8. Mayorga TJ. 2015. Estrés oxidativo: ¿un estado celular defectuoso para la función espermática?. Rev Chil Obstet Ginecol 80: 486-492.

9. Ortega FC. 2011. Factores implicados en la variabilidad individual en la respuesta a la congelación del eyaculado equino: estructura de subpoblaciones, estrés oxidativo y cambios apoptóticos. Tesis doctoral, Fac.Vet.Univ Extremadura, España, p.1-176.

10. Palma C, Vinay BJ. 2014. Infertilidad masculina. Rev Med Clin Las Condes 25: 122-128.

11. Paparella CV, Pavesi AB, Feldman RN, Bouvet BR. 2015. Importancia de la evaluación del estrés oxidativo en el semen humano. Arch Med Interna 37: 7-14.

12. Thongrueang N, Chaibangyang N, Chanapiwat P, Kaeoket K. 2017. Effects of adding melatonin on the quality of frozen-thawed boar semen. J Appl Anim Sci 10: 47-56.

13. Villa NA, Castaño D, Duque PC, Ceballos A. 2012. Glutatione peroxidase and superoxide dismutase activities in blood and seminal plasma in colombian stallions. Rev Colomb Cienc Pecu 25: 64-70.

14. Yeste M, Rodríguez GJ, Bonet S. 2017. Artificial insemination with frozen-thawed boar sperm. Molec Reprod Dev 2017: 1-12. 